\title{
Non-Commutative Chaotic Expansion of Hilbert-Schmidt Operators on Fock Space
}

\author{
Stéphane Attal \\ Institut de Recherche Mathématique Avancée, Université Louis Pasteur et C.N.R.S., \\ 7, rue René Descartes, F-67084 Strasbourg Cedex, France
}

Received: 21 February 1994/in revised form: 17 October 1994

\begin{abstract}
It is known, from a simple algebraic computation, that every HilbertSchmidt operator on the Fock space admits a Maassen-Meyer kernel. MaassenMeyer kernels are a non-commutative extension of the usual notion of chaotic expansion of random variables. Using an extension of the non-commutative stochastic integrals which allows to define these integrals on the whole Fock space, we prove that a Hilbert-Schmidt operator on Fock space is the sum of a series of iterated non-commutative stochastic integrals with respect to the basic three quantum noises. In this way we recover its Maassen-Meyer kernel which can be completely described from the operator itself.
\end{abstract}

\section{Introduction}

It is well-known that every square integrable functional $f$ of the Wiener process $\left(W_{t}\right)_{t \geqq 0}$ admits a previsible representation, that is a representation as the sum of a constant (its expectation) and a stochastic integral of a previsible process with respect to $W$. But such a random variable also admits a chaotic expansion [7], that is, a representation of the form

$$
f=\mathbb{E}[f]+\sum_{n=1}^{\infty} \int_{0<t_{1}<\cdots<t_{n}} f_{n}\left(t_{1}, \ldots, t_{n}\right) d W_{t_{1}} \cdots d W_{t_{n}},
$$

where $f_{n}$ is a square integrable function on the increasing simplex

$$
\Sigma_{n}=\left\{\left(t_{1}, \ldots, t_{n}\right) \in \mathbb{R}^{n}, 0<t_{1}<\cdots<t_{n}\right\} .
$$

The set $\mathscr{P}_{n}$ of subsets of $\mathbb{R}^{+}$with cardinality $n$ can be clearly identified to $\Sigma_{n}$. The family $\left\{f_{n}\right\}$ can be viewed as a single square integrable function $\widehat{f}$ on $\mathscr{P}=$ $\bigcup_{n} \mathscr{P}_{n}\left(\mathscr{P}_{0}=\{\emptyset\}\right)$, by putting $\widehat{f}(A)=f_{n}\left(t_{1}, \ldots, t_{n}\right)$ if $A=\left\{0<t_{1}<\cdots<t_{n}\right\} \in \mathscr{P}$, with the convention $\widehat{f}(\emptyset)=\mathbb{E}[f]$. With this "short notation" ([3]) the chaotic expansion of $f$ can be written $f=\int_{\mathscr{P}} \widehat{f}(A) d W_{A}$.

On the boson Fock space $\Phi$ over $L^{2}\left(\mathbb{R}^{+}\right)$, which is isomorphic to the space of square integrable Wiener functionals $([15])$, operators can be represented in two 
ways. Following Hudson and Parthasarthy's theory [6], some of them can be represented as a sum of non-commutative stochastic integrals of adapted processes of operators integrated with respect to the three basic quantum noises: the creation, annihilation and gauge processes, respectively denoted $\left(A_{t}^{+}\right)_{t \geqq 0},\left(A_{t}^{-}\right)_{t \geqq 0}$ and $\left(A_{t}^{\circ}\right)_{t \geqq 0}$. This representation can be seen as a quantum extension of the previsible representation of random variables.

The second kind of representation of operators on the Fock space $\Phi$, is the representation by Maassen-Meyer kernels, defined under their first form with two arguments by Maassen [9] and under their definitive form with three arguments by Meyer [11]. Maassen-Meyer kernels are to the non-commutative stochastic calculus as what chaotic expansions are to the classical one. That is, a Maassen-Meyer kernel is formally an operator $T$ on $\Phi$ which admits a representation as a series of iterated non-commutative stochastic integrals of scalar operators with respect to the creation, annihilation and gauge processes. Using the same kind of "short notation" as before, this can be written

$$
T=\int_{\mathscr{P} 3} \widehat{T}(A, B, C) d A_{A}^{+} d A_{B}^{\circ} d A_{C}^{-},
$$

where the subsets $A, B$ and $C$ can be supposed disjoint.

This has no rigorous meaning, in particular this form no longer respects the adaptedness of the integrated processes and the convergence of the series has to be studied. But one can formally describe how such an operator acts on a vector of the Fock space. It suffices to determine the action of an operator of the form $d A_{A}^{+} d A_{B}^{\circ} d A_{C}^{-}$on a basis continuous element of the Wiener chaos $d W_{L}$. One obtains (cf. [10]) that the image $T f$ of $f$ under $T$ has for coefficients in its chaotic expansion

$$
\widehat{T f}(A)=\int_{\mathscr{P}} \sum_{U+V+W=A} \widehat{T}(U, V, M) \widehat{f}(M+V+W) d M .
$$

Although this is not rigorous, the latter identity is for some "good" operators and some "good" vectors. This is the rigorous definition of Maassen and Meyer.

In this paper we study Hilbert-Schmidt operators on the boson Fock space $\Phi$. We first recall a result of [8] which proves by an algebraic computation that they admit a Maassen-Meyer kernel. The aim of the paper is to prove that this MaassenMeyer kernel really corresponds to a non-commutative chaotic expansion.

Recall that the chaotic expansion property of square integrable Wiener functionals can be proved by iterating the previsible representation. Indeed, every element $f$ of $L^{2}(\Omega, \mathscr{F}, P)$ can be written $f=\mathbb{E}[f]+\int_{0}^{\infty} \psi_{s} d W_{s}$, where $\psi$ is a previsible process in $L^{2}(\Omega, \mathscr{F}, P)$. So, for almost all $s, \psi_{s}$ is an element of $L^{2}(\Omega, \mathscr{F}, P)$. Then one has $\psi_{s}=\mathbb{E}\left[\psi_{s}\right]+\int_{0}^{s} \psi_{s, u}^{\prime} d W_{u}$, for a previsible process $\left(\psi_{s, u}^{\prime}\right)_{u \leqq s}$. Inserting this identity in the representation of $f$, one obtains

$$
f=\mathbb{E}[f]+\int_{0}^{\infty} \mathbb{E}\left[\psi_{s}\right] d W_{s}+\int_{0}^{t} \int_{0}^{s} \psi_{s, u}^{\prime} d W_{u} d W_{s}
$$

One can iterate this operation arbitrarily many times. One then obtains two terms in the representation of $f$ : a sum of iterated stochastic integrals of deterministic functions on the simplexes and an iterated stochastic integral of a previsible process indexed by a simplex. The first term constitutes the beginning of the chaotic expansion of $f$, the second term disappears when one iterates the procedure. 
In this paper we apply the same idea to the non-commutative case. That is, using an extension of the notion of non-commutative stochastic integrals [2], we prove that every Hilbert-Schmidt operator on the Fock space admits an extended integral representation, with respect to the three basic quantum noises, valued on all the Fock space. We show that this representation can be iterated arbitrarily many times. In this way, we obtain a sum of iterated non-commutative stochastic integrals of scalar operators which constitutes the beginning of a non-commutative chaotic expansion of the operator, and another term which we prove converges to 0 in a certain sense. We show that from this chaotic expansion one recovers the Maassen-Meyer kernel. In this way, we obtain a description of the kernel in terms of the operator itself; this is a justification to the previous non-rigorous presentation of these kernels.

There exists earlier literature about Hilbert-Schmidt operators and quantum stochastic calculus on Fock space ([4,5 and 8]). The fact that Hilbert-Schmidt operators on the Fock space admit a Maassen-Meyer kernel was already observed. The difference with this article is that here we want to apply to this case a new method for finding operators admitting a Maassen-Meyer kernel. This idea is based on the iteration of the integral representation procedure. This method, copied from the classical stochastic calculus, consists in the following: each time one has a "good" family of operators on the Fock space, admitting a non-commutative integral representation such that the coefficients of this representation belong to the same family, one can iterate arbitrarily many times this integral representation. If one has good estimates, we can see that the constant terms of each integral representation will, at the end, give the "non-commutative chaotic expansion" of the operator, while the remainder will vanish. Then an algebraic computation will give the Maassen-Meyer kernel of the operator. The aim of this article is to exploit the fact that the family of Hilbert-Schmidt operators is such a good family. We apply our procedure to it. This gives the announced results, and the hope that one can apply this method to other families of operators.

\section{Notations}

Let $(\Omega, \mathscr{F}, P)$ be the Wiener space. Let $\left(W_{t}\right)_{t \geqq 0}$ be the canonical Brownian motion on $\Omega$. For every $t \in \mathbb{R}^{+}$, let $\mathscr{F}_{t]}$, resp. $\mathscr{F}_{[t}$ be the $\sigma$-field generated by $\left\{W_{u} ; u \leqq t\right\}$, resp. $\left\{W_{u}-W_{t} ; u \geqq t\right\}$.

Let $\Phi, \Phi_{t]}$ and $\Phi_{[t}$ be the symmetric Fock spaces over respectively $L^{2}\left(\mathbb{R}^{+}\right)$, $L^{2}([0, t])$ and $L^{2}([t,+\infty[)$.

We then have the following identifications [11]:

$$
\left\{\begin{array}{l}
\Phi \simeq L^{2}(\Omega, \mathscr{F}, P) \\
\Phi_{t]} \simeq L^{2}\left(\Omega, \mathscr{F}_{t]}, P\right) \\
\Phi_{[t} \simeq L^{2}\left(\Omega, \mathscr{F}_{[t}, P\right) .
\end{array}\right.
$$

The orthogonal projection from $\Phi$ onto $\Phi_{t]}$ is denoted by $\mathbb{E}_{t}$ (it can be interpreted as the operator of conditional expectation with respect to $\left.\mathscr{F}_{t}\right)$. For all $u \in L^{2}\left(\mathbb{R}^{+}\right)$, let $\varepsilon(u)$ be the stochastic exponential at the infinity of the martingale $\int_{0}^{*} u(s) d W_{s}$. Recall that $\varepsilon(u)$ is, in the Fock space structure, the coherent vector associated to $u$. 
For all $s \leqq t$, let $u_{t]}=u \mathbb{1}_{[0, t]}, \quad u_{[t}=u \mathbb{1}_{[t,+\infty}$. Recall that the random variable $\varepsilon\left(u_{t]}\right)$ admits the previsible representation $\varepsilon\left(u_{t]}\right)=1+\int_{0}^{t} u(s) \varepsilon\left(u_{s]}\right) d W_{s}$. So

$$
\left\langle\varepsilon\left(u_{t]}\right), \varepsilon\left(v_{t]}\right)\right\rangle=\exp \left(\int_{0}^{t} \bar{u}(s) v(s) d s\right), \quad \text { for all } u, v \in L^{2}\left(\mathbb{R}^{+}\right) .
$$

Let $L_{l b}^{2}\left(\mathbb{R}^{+}\right)$be the subspace of locally bounded elements of $L^{2}\left(\mathbb{R}^{+}\right)$. Denote by $\mathscr{E}_{l b}$ the space of finite linear combinations of vectors $\varepsilon(u)$, for $u \in L_{l b}^{2}\left(\mathbb{R}^{+}\right)$. Recall that this subspace is dense in $\Phi$.

\section{Some Elements of Non-Commutative Stochastic Calculus}

We present here only what is needed in the paper. For a more complete presentation of the subject one should refer to the original paper of Hudson and Parthasarathy [6], to Meyer's book [10] or Parthasarathy's book [13].

Note that all that is written in this article depends only on the structure of Fock space. We will discuss this point in Sect. 4.

Recall that the boson Fock space $\Phi$ has a "continuous tensor product structure." That is, for all $t \in \mathbb{R}^{+}, \Phi \simeq \Phi_{t]} \otimes \Phi_{[t}$. Recall that, in this structure, exponential vectors are homogeneous elements: $\varepsilon(u)=\varepsilon\left(u_{t]}\right) \otimes \varepsilon\left(u_{[t}\right)$. In the following we omit the tensor product symbol.

A family of operators $\left(H_{t}\right)_{t \geqq 0}$ from $\Phi$ into $\Phi$, defined on $\mathscr{E}_{l b}$, is an adapted process of operators if, for all $u \in L_{l b}^{2}\left(\mathbb{R}^{+}\right)$, the mapping $t \mapsto H_{t} \varepsilon\left(u_{t]}\right)$ is strongly measurable and if, for all $t \in \mathbb{R}^{+}$,

$$
\left\{\begin{array}{l}
H_{t} \varepsilon\left(u_{t]}\right) \in \Phi_{t]} \\
H_{t} \varepsilon(u)=\left[H_{t} \varepsilon\left(u_{t]}\right)\right] \varepsilon\left(u_{[t}\right) .
\end{array}\right.
$$

That is $H_{t}=H_{t \mid \Phi_{t]}} \otimes I_{\mid \Phi_{[t}}$ in the structure $\Phi \simeq \Phi_{t]} \otimes \Phi_{[t}$.

An adapted process of operators $\left(M_{t}\right)_{t \geqq 0}$ is a martingale of operators if, for all $s \leqq t, u, v \in L_{l b}^{2}\left(\mathbb{R}^{+}\right),\left\langle\varepsilon\left(u_{s]}\right), M_{t} \varepsilon\left(v_{s]}\right)\right\rangle=\left\langle\varepsilon\left(u_{s]}\right), M_{s} \varepsilon\left(v_{s]}\right)\right\rangle$, that is

$$
\mathbb{E}_{s} M_{t} \mathbb{E}_{s}=\mathbb{E}_{s} M_{s} \mathbb{E}_{s}\left(=M_{s} \mathbb{E}_{s}\right) .
$$

If $T$ is an operator on $\Phi$, defined on $\mathscr{E}_{l b}$, the family $\left(T_{t}\right)_{t \geqq 0}$ of operators, defined by

$$
T_{t} \varepsilon(u)=\left[\mathbb{E}_{t} T \varepsilon\left(u_{t]}\right)\right] \varepsilon\left(u_{[t}\right), \quad t \in \mathbb{R}^{+}, u \in L_{l b}^{2}\left(\mathbb{R}^{+}\right),
$$

is a martingale of operators, called the martingale associated with $T$.

The three particular martingales of creation, annihilation and gauge, will be respectively denoted $\left(A_{t}^{+}\right)_{t \geqq 0},\left(A_{t}^{-}\right)_{t \geqq 0},\left(A_{t}^{\circ}\right)_{t \geqq 0}$; they verify

$$
\begin{aligned}
& \left\langle\varepsilon(u), A_{t}^{+} \varepsilon(v)\right\rangle=\langle\varepsilon(u), \varepsilon(v)\rangle \int_{0}^{t} \bar{u}(s) d s, \\
& \left\langle\varepsilon(u), A_{t}^{-} \varepsilon(v)\right\rangle=\langle\varepsilon(u), \varepsilon(v)\rangle \int_{0}^{t} v(s) d s, \\
& \left\langle\varepsilon(u), A_{t}^{\circ} \varepsilon(v)\right\rangle=\langle\varepsilon(u), \varepsilon(v)\rangle \int_{0}^{t} \bar{u}(s) v(s) d s,
\end{aligned}
$$


for all $t \in \mathbb{R}^{+}, u, v \in L_{l b}^{2}\left(\mathbb{R}^{+}\right)$. These identities a priori only define quadratic forms on the Fock space, but they are proved to define operators on $\mathscr{E} l b$ in [6]. The same remark holds for the identity (3) below.

If $H^{\circ}, H^{+}, H^{-}$are adapted processes of operators verifying, for all $u \in L_{l b}^{2}\left(\mathbb{R}^{+}\right)$, all $t \in \mathbb{R}^{+}$,

$$
\int_{0}^{t}|u(s)|\left\|H_{s}^{-} \varepsilon(u)\right\|+\left\|H_{s}^{+} \varepsilon(u)\right\|^{2}+|u(s)|^{2}\left\|H_{s}^{\circ} \varepsilon(u)\right\|^{2} d s<\infty,
$$

then the family of non-commutative stochastic integrals

$$
T_{t}=\int_{0}^{t} H_{s}^{\circ} d A_{s}^{\circ}+\int_{0}^{t} H_{s}^{-} d A_{s}^{-}+\int_{0}^{t} H_{s}^{+} d A_{s}^{+}, \quad t \in \mathbb{R}^{+},
$$

is well defined on $\mathscr{E}_{l b}$ as a martingale of operators verifying, for all $t \in \mathbb{R}^{+}$, and all $u, v \in L_{l b}^{2}\left(\mathbb{R}^{+}\right)$,

$$
\left\langle\varepsilon(u), T_{t} \varepsilon(v)\right\rangle=\int_{0}^{t}\left\langle\varepsilon(u), \bar{u}(s) v(s) H_{s}^{\circ} \varepsilon(v)+v(s) H_{s}^{-} \varepsilon(v)+\bar{u}(s) H_{s}^{+} \varepsilon(v)\right\rangle d s .
$$

Remark. 1 . If the adjoint processes $\left(H^{\varepsilon}\right)^{*}$ are also defined on $\mathscr{E}_{l b}$ and if they verify

$$
\int_{0}^{t}\left(\left|u(s)\|\|\left(H_{s}^{+}\right)^{*} \varepsilon(u)\|+\|\left(H_{s}^{-}\right)^{*} \varepsilon(u)\left\|^{2}+|u(s)|^{2}\right\|\left(H_{s}^{\circ}\right)^{*} \varepsilon(u) \|^{2}\right) d s<\infty,\right.
$$

then the process $\left(T_{t}^{*}\right)_{t \geqq 0}$ admits the integral representation

$$
T_{t}^{*}=\int_{0}^{t}\left(H_{s}^{\circ}\right)^{*} d A_{s}^{\circ}+\int_{0}^{t}\left(H_{s}^{+}\right)^{*} d A_{s}^{-}+\int_{0}^{t}\left(H_{s}^{-}\right)^{*} d A_{s}^{+} .
$$

Remark. 2. The non-commutative stochastic integral $T_{t}$, can be defined for $t=+\infty$ in the same way if (1) is verified for $t=+\infty$.

For all $\varepsilon \in\{+, \circ,-\}$, the martingale associated with the operator $\int_{0}^{\infty} H_{s}^{\varepsilon} d A_{s}^{\varepsilon}$ is $\left(\int_{0}^{t} H_{s}^{\varepsilon} d A_{s}^{\varepsilon}\right)_{t \geqq 0}$.

In the following, each time one of these integrals appears we assume that the processes $H^{\circ}, H^{+}$and $H^{-}$verify (1).

\section{Extension of the Non-Commutative Stochastic Integrals}

Let us suppose that $T$ is a bounded operator on $\Phi$ such that, on $\mathscr{E} l b$,

$$
T=\int_{0}^{\infty} H_{s}^{\circ} d A_{s}^{\circ}+\int_{0}^{\infty} H_{s}^{-} d A_{s}^{-}+\int_{0}^{\infty} H_{s}^{+} d A_{s}^{+},
$$

where $\mathrm{H}^{\circ}, \mathrm{H}^{-}$and $\mathrm{H}^{+}$are bounded operator processes. The definition (2) of these integrals only defines them on the domain $\mathscr{E} l b$. As we want to study bounded operators, we use the extension of the notion of non-commutative stochastic integral given in [2].

We first need a little discussion to see that, despite the notations, the following (and the entire article) is intrinsic to the Fock space structure. Let $\mathscr{P}$ be 
the set of finite subsets of $\mathbb{R}^{+}$. As $\mathscr{P}=\bigcup_{n} \mathscr{P}_{n}$, where $\mathscr{P}_{0}=\{\emptyset\}$, and $\mathscr{P}_{n}$ is the set of $n$-element subsets of $\mathbb{R}^{+}, n \in \mathbb{N}^{*}$, as each $\mathscr{P}_{n}$ can be identified to a subset of $\mathbb{R}^{n}, \mathscr{P}$ acquires a natural measure denoted $d A(A \in \mathscr{P})$. One knows ([3]) that the Fock space $\Phi$ is isomorphic to the Guichardet space $L^{2}(\mathscr{P})$; so every element $f$ of $\Phi$ is determined by its chaotic expansion, that is a square integrable function $\widehat{f}$ on $\mathscr{P}$. Furthermore, one has $\|f\|^{2}=\int_{\mathscr{P}}|\widehat{f}(A)|^{2} d A$. For each $f \in \Phi$ one can define a family $\left(\dot{f}_{s}\right)_{s \geqq 0}$ such that, for almost all $s, \dot{f}_{s}$ is an element of $\Phi$ whose

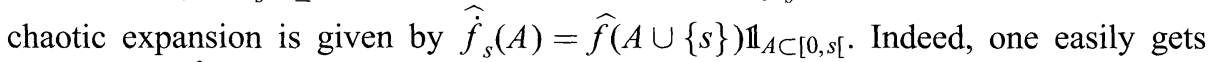
that $\int_{0}^{\infty} \int_{\mathscr{P}}\left|\widehat{\dot{f}}_{s}(A)\right|^{2} d A d s<\infty$. For any family $\left(g_{t}\right)_{t \geqq 0}$ made of elements of $\Phi$ such that $g_{t} \in \Phi_{t]}$ and $\int_{0}^{\infty}\left\|g_{t}\right\|^{2} d t<\infty$, one can define a new element of $\Phi$, denoted $I(g)$ or $\int_{0}^{\infty} g_{t} d W_{t}$, such that $\widehat{I(g)}(A)=0$ if $A=\emptyset$ and $\widehat{I(g)}(A)=\widehat{g_{\vee A}}(A-)$ otherwise (where $\vee A=\max A$ and $A-=A \backslash\{\vee A\}$ ). Apply that to the family $\left(\dot{f}_{t}\right)_{t \geqq 0}$; one gets easily that $\widehat{I(\dot{f})}(A)=\widehat{f}(A)$ if $A \neq \emptyset, 0$ otherwise. Denote $\mathbb{E}[f]$ the element of $\Phi$ such that $\widehat{\mathbb{E}[f]}(A)=\widehat{f}(\emptyset)$ if $A=\emptyset, 0$ otherwise. One finally gets that, for every $f \in \Phi, f$ admits a representation, called previsible representation of $f$, of the form $f=\mathbb{E}[f]+\int_{0}^{\infty} \dot{f}_{s} d W_{s}$, with $\|f\|^{2}=\mathbb{E}[f]^{2}+\int_{0}^{\infty}\left\|\dot{f}_{s}\right\|^{2} d s$. Of course, in a probabilistic interpretation of $\Phi$ this representation is really the previsible representation of $f$, but as we have seen, it is a completely intrinsic object. We can now present the results of [2], without worrying about the notations.

Let $f_{t}=\mathbb{E}_{t} f, f_{\infty}=f$ and

$$
T_{t}=\int_{0}^{t} H_{s}^{\circ} d A_{s}^{\circ}+\int_{0}^{t} H_{s}^{-} d A_{s}^{-}+\int_{0}^{t} H_{s}^{+} d A_{s}^{+}, \quad t \in \overline{\mathbb{R}}_{+} .
$$

It is proved in [2] that, if $f$ is in the space $\mathscr{E}_{l b}$, we have, for all $t \in \overline{\mathbb{R}}_{+}$,

$$
T_{t} f_{t}=\int_{0}^{t} T_{s} \dot{f_{s}} d W_{s}+\int_{0}^{t} H_{s}^{\circ} \dot{f_{s}} d W_{s}+\int_{0}^{t} H_{s}^{-} \dot{f_{s}} d s+\int_{0}^{t} H_{s}^{+} f_{s} d W_{s}
$$

On $\mathscr{E}_{l b}$, this equation is equivalent to (2) ([2], Theorem 1), but it has the advantage of having a meaning for all $f$ in $\Phi$.

Definition. Let $\left(T_{t}\right)_{t \geqq 0}$ be a martingale of bounded operators on $\Phi$, let $H^{\circ}, H^{-}$ and $\mathrm{H}^{+}$be adapted processes of bounded operators, we say that

$$
T_{t}=\int_{0}^{t} H_{s}^{\circ} d A_{s}^{\circ}+\int_{0}^{t} H_{s}^{-} d A_{s}^{-}+\int_{0}^{t} H_{s}^{+} d A_{s}^{+}, \quad t \geqq 0
$$

in the extended sense on all $\Phi$, if (4) is well defined and true for all $f \in \Phi$.

Theorem 4.1 ([2], Theorem 2).Let $\left(T_{t}\right)_{t \geqq 0}$ be an adapted process of operators such that the processes $\left(T_{t}\right)_{t \geqq 0}$ and $\left(T_{t}^{*}\right)_{t \geqq 0}$ admit an integral representation on $\mathscr{E}_{l b}$. Then both integral representations can be extended wherever it is meaningful. 


\section{Regular Martingales}

Parthasarathy and Sinha [14] have defined a class of regular martingales. A martingale of operators $\left(T_{t}\right)_{t \geqq 0}$ is said to be regular if there exists a Radon measure $m$ on $\mathbb{R}^{+}$such that, for all $f \in \mathscr{E}_{l b}$, with associated martingale $\left(f_{t}\right)_{t \geqq 0}$, for every $r<s<t$, one has

$$
\begin{aligned}
\left\|T_{t} f_{r}-T_{s} f_{r}\right\|^{2} & \leqq\left\|f_{r}\right\|^{2} m([s, t]), \\
\left\|T_{t}^{*} f_{r}-T_{s}^{*} f_{r}\right\|^{2} & \leqq\left\|f_{r}\right\|^{2} m([s, t]) .
\end{aligned}
$$

We now recall the Parthasarathy-Sinha theorem ([14]).

Theorem 5.1. Let $\left(T_{t}\right)_{t \geqq 0}$ be a martingale of bounded operators on $\Phi$. The following two statements are equivalent.

i) There exists $\lambda \in \mathbb{C}$ and three bounded adapted processes $\mathrm{H}^{\circ}, \mathrm{H}^{-}$and $\mathrm{H}^{+}$ such that, on $\mathscr{E}_{l b}$,

$$
T_{t}=\lambda I+\int_{0}^{t} H_{s}^{\circ} d A_{s}^{\circ}+\int_{0}^{t} H_{s}^{-} d A_{s}^{-}+\int_{0}^{t} H_{s}^{+} d A_{s}^{+},
$$

where $\left\|H .^{-}\right\|$and $\left\|H .^{+}\right\|$are locally square integrable functions.

ii) The martingale $\left(T_{t}\right)_{t \geqq 0}$ is regular with respect to some Radon measure $m$ on $\mathbb{R}^{+}$.

Under these assumptions we have $\max \left\{\left\|H_{s}^{-}\right\|^{2},\left\|H_{s}^{+}\right\|^{2}\right\} \leqq m^{\prime}(s)$, a.a. s, where $m^{\prime}$ denotes the derivative of the absolutely continuous part of $m$.

We recall a useful remark of Meyer [12]. If $\left(T_{t}\right)_{t \geqq 0}$ is a regular martingale the coefficient $H .^{\circ}$ of $\left(A_{t}^{\circ}\right)_{t \geqq 0}$, appearing in the integral representation of $T_{t}$, has a locally bounded norm.

So, by the extension Theorem 4.1, we have that the integral representation of any regular martingale can be extended to all of $\Phi$ (cf. [1], Corollary 2, for more details).

\section{Maassen-Meyer Kernels}

We now recall the definition of Maassen-Meyer kernels (see [9 and 10] for details). An operator $T$ from Fock space $\Phi$ to itself is said to have a Maassen-Meyer kernel, if there exists a set-function $\widehat{T}$ on $\mathscr{P}^{3}$ (in fact $\widehat{T}$ needs to be defined only on pairwise disjoint $A, B, C$ in $\mathscr{P}^{3}$ ) such that, for "sufficiently many" vectors $f$ of $\Phi$, one has

$$
\widehat{T f}(A)=\int_{\mathscr{P}} \sum_{U+V+W=A} \widehat{T}(U, V, M) \widehat{f}(M+V+W) d M,
$$

where the symbol "+" denotes an union of disjoint elements of $\mathscr{P}$.

In Maassen's theory supposing the kernels bounded implies (5) is well-defined for all elements of a class of "test-vectors." But this part of Maassen's theory has nothing to do with our purpose. We just need the definition of the class of "test- 
vectors." An element $f$ of $\Phi$, with chaotic expansion

$$
f=\int_{A \in \mathscr{P}} \widehat{f}(A) d W_{A}
$$

is a test-vector if it verifies a compact time support condition: $\widehat{f}(A)=0$ unless $A$ is included in some $[0, T]$, and a domination condition: $|\widehat{f}(A)| \leqq C^{|A|}$, where $|A|$ denotes the cardinal of $A$.

\section{Maassen-Meyer Kernels of Hilbert-Schmidt Operators}

The starting point of this work is the following result, already proved in [8].

Theorem 7.1. Let $H$ be an Hilbert-Schmidt operator from $\Phi$ into $\Phi$; then there exists a mapping $\widehat{H}$ from $\mathscr{P}^{3}$ into $\mathbb{R}$ such that, for all $f \in \Phi$, all $A \in \mathscr{P}$, we have

$$
\widehat{H f}(A)=\int_{\mathscr{P}} \sum_{U+V+W=A} \widehat{H}(U, V, M) \widehat{f}(M+V+W) d M .
$$

Proof. Let $H$ be an Hilbert-Schmidt operator from $\Phi$ into $\Phi$. As $\Phi$ is isomorphic to the space $L^{2}(\mathscr{P})$, where $\mathscr{P}$ is endowed with the $\sigma$-finite measure described previously, $H$ then is an Hilbert-Schmidt operator from $L^{2}(\mathscr{P})$ into $L^{2}(\mathscr{P})$. Therefore, $H$ admits a kernel representation, that is there exists a mapping $\varphi$ from $\mathscr{P}^{2}$ into $\mathbb{R}$ such that

$$
\int_{\mathscr{P}^{2}} \varphi(A, B)^{2} d A d B<\infty
$$

and such that, for all $f \in L^{2}(\mathscr{P})$,

$$
\widehat{H f}(A)=\int_{\mathscr{P}} \varphi(A, M) f(M) d M .
$$

Put $\widehat{H}(A, B, C)=(-1)^{|B|} \varphi(A, C)$. One has

$$
\widehat{H f}(A)=\int_{\mathscr{P}} \sum_{U \subset A} \mathbb{1}_{\{A \backslash U=\emptyset\}} \varphi(U, M) f(M+A \backslash U) d M .
$$

The Möbius inversion formula gives, for all $C \in \mathscr{P}$,

$$
\sum_{V \subset C}(-1)^{|V|}=\mathbb{1}_{\{C=\emptyset\}}
$$

so one finally gets

$$
\begin{aligned}
\widehat{H f}(A) & =\int_{\mathscr{P}} \sum_{U \subset A} \sum_{V \subset A \backslash U}(-1)^{|V|} \varphi(U, M) f(M+A \backslash U) d M \\
& =\int_{\mathscr{P}} \sum_{U \subset A} \sum_{V \subset A \backslash U} \widehat{H}(U, V, M) f(M+A \backslash U) d M \\
& =\int_{\mathscr{P}} \sum_{U+V+W=A} \widehat{H}(U, V, M) f(M+V+W) d M .
\end{aligned}
$$


This simple result indicates that every Hilbert-Schmidt operator from $\Phi$ into $\Phi$ admits a Maassen-Meyer kernel. The main result of the following section is that, in the case of Hilbert-Schmidt operators, this kernel really corresponds to a series of multiple reordered non-commutative stochastic integrals, in a rigourous sense.

\section{Iteration of the Previsible Representation}

We are going to forget Theorem 7.1 for a moment and try to apply, in the noncommutative context, the previsible representation iteration procedure. That is, we are going to prove that one can iterate infinitely many times the representation of Hilbert-Schmidt operators as non-commutative stochastic integrals.

Let us first detail some notations and preliminaries.

A martingale $\left(H_{t}\right)_{t \geqq 0}$ of operators from $\Phi$ into $\Phi$ is an Hilbert-Schmidt martingale if, for all $t \in \mathbb{R}^{+}$, the operator $H_{t}$, restricted to $\Phi_{t]}$, is an Hilbert-Schmidt operator.

We denote $\|\cdot\|_{H S}$ the Hilbert-Schmidt norm of operators.

For all $s \leqq t$, we denote by $\Phi_{s]}^{\perp t]}$ the orthogonal of $\Phi_{s]}$ into $\Phi_{t]}$ and $\Phi_{t]}^{\perp}$ the orthogonal of $\Phi_{t]}$ into $\Phi$.

We sometimes use the notation $\Phi_{\infty}$ for $\Phi$.

Lemma 8.1. Let $t$ be fixed in $[0, \infty]$, let $H_{t}$ be an Hilbert-Schmidt operator from $\Phi_{t]}$ into $\Phi_{t]}$. Then, for all $s<t$, the operator $\mathbb{E}_{s} H_{t}$ is an Hilbert-Schmidt operator from $\Phi_{s]}$ into $\Phi_{s]}$.

Proof. One has $\Phi_{t]}=\Phi_{s]} \oplus \Phi_{s]}^{\perp t]}$. Let $\left(u^{n}\right)_{n \in \mathbb{N}}$, resp. $\left(v^{n}\right)_{n \in \mathbb{N}}$, be an o.n.b. of $\Phi_{s]}$, resp. $\Phi_{s]}^{\perp t]}$. The family $\left(w^{n}\right)_{n \in \mathbb{N}}$, defined by $w^{2 n}=u^{n}, w^{2 n+1}=v^{n}$ then is an o.n.b. of $\Phi_{t]}$. Thus, one has

$$
\begin{aligned}
\sum_{n=0}^{\infty}\left\|\mathbb{E}_{S} H_{t} u^{n}\right\|^{2} & \leqq \sum_{n=0}^{\infty}\left\|H_{t} u^{n}\right\|^{2} \\
& \leqq \sum_{n=0}^{\infty}\left\|H_{t} u^{n}\right\|^{2}+\sum_{n=0}^{\infty}\left\|H_{t} v^{n}\right\|^{2} \\
& =\sum_{n=0}^{\infty}\left\|H_{t} w^{n}\right\|^{2}=\|H\|_{H S}^{2}<\infty
\end{aligned}
$$

In Parthasarathy and Sinha's article [14], it is proved that every Hilbert-Schmidt martingale is a regular martingale. By Theorem 5.1 and the remarks which follow it, every Hilbert-Schmidt martingale is representable as a sum of non-commutative stochastic integrals in the extended sense on all $\Phi$ (note that the results of the non-commutative integral representation of Hilbert-Schmidt operators on the exponential domain was first proved in [5], but the language of regular martingales was not yet used). We subsume these results, and an easy extension, in the following proposition.

Proposition 8.2. Let $t \in[0,+\infty]$ be fixed, let $H_{t}$ be an Hilbert-Schmidt operator from $\Phi_{t]}$ into $\Phi_{t]}$. Then $H_{t}$ admits a non-commutative stochastic integral 
representation, in the extended sense on all $\Phi$, of the form

$$
H_{t}=\mathbb{E}\left[H_{t} \mathbb{1}\right] I-\int_{0}^{t} H_{s}^{\circ} d A_{s}^{\circ}+\int_{0}^{t} H_{s}^{-} d A_{s}^{-}+\int_{0}^{t} H_{s}^{+} d A_{s}^{+},
$$

where $\left(H_{s}^{\circ}\right)_{s \leqq t}$ is the martingale associated to $H_{t}$, and where, for all $\varepsilon=-,+$, for almost all $s \leqq t, H_{s}^{\varepsilon}$ is an Hilbert-Schmidt operator from $\Phi_{s]}$ into $\Phi_{s]}$ such that

$$
\int_{0}^{t}\left\|H_{s}^{\varepsilon}\right\|_{H S}^{2} d s \leqq\left\|H_{t}\right\|_{H S}^{2} .
$$

Proof.

$1^{\text {st }}$ case: $t=+\infty$. If $H$ is an Hilbert-Schmidt operator from $\Phi$ into $\Phi$ then, by Lemma 8.1 , the martingale associated to $\left(H_{t}^{\circ}\right)_{t \geqq 0}$ is an Hilbert-Schmidt martingale. In [14], Proposition 4.3 and Theorem 4.4, it is proved that $\left(H_{t}^{\circ}\right)_{t \geqq 0}$ is then a regular martingale, so it is representable on all $\Phi$. But, it is also proved in [14] that the integral representation has the form

$$
H_{t}^{\circ}=H_{0}^{\circ}-\int_{0}^{t} H_{s}^{\circ} d A_{s}^{\circ}+\int_{0}^{t} H_{s}^{-} d A_{s}^{-}+\int_{0}^{t} H_{s}^{+} d A_{s}^{+},
$$

where it must be remarked that the coefficient of $d A^{\circ}$ is, up to a sign, the martingale $\left(H_{t}^{\circ}\right)_{t \geqq 0}$ itself. Furthermore, it is proved in the same reference that, for almost all $s \in \mathbb{R}^{+}$, all $\varepsilon \in\{-,+\}, H_{s}^{\varepsilon}$ is an Hilbert-Schmidt operator from $\Phi_{s]}$ into $\Phi_{s]}$ and that, if one defines $\alpha_{H}(t)=\left\|H_{t}^{\circ}\right\|_{H S}^{2}, t \in \overline{\mathbb{R}^{+}}$, then $\alpha_{H}$ in a non-decreasing continuous function, which verifies $\left\|H_{t}^{\varepsilon}\right\|_{H S}^{2} \leqq \alpha_{H}^{\prime}(s)$, where $\alpha_{H}^{\prime}$ is the absolutely continuous part of the Stieltjes measure associated to $\alpha_{H}$. So

$$
\int_{0}^{\infty}\left\|H_{t}^{\varepsilon}\right\|_{H S}^{2} d s \leqq \alpha_{H}(\infty)-\alpha_{H}(0) \leqq \alpha_{H}(\infty)=\|H\|_{H S}^{2} .
$$

$2^{\text {nd }}$ case: $t<+\infty$. Let $H_{t}$ be an Hilbert-Schmidt operator from $\Phi_{t]}$ into $\Phi_{t]}$ and $\left(H_{S}^{\circ}\right)_{S \leqq t}$ be the associated martingale. By Lemma 8.1, it is an Hilbert-Schmidt martingale but indexed only by $[0, t]$, so one cannot apply Parthasarathy and Sinha theorem directly. One must extend $\left(H_{s}^{\circ}\right)_{s \leqq t}$ into an Hilbert-Schmidt martingale indexed by $\mathbb{R}^{+}$.

Put $H_{s}^{\circ}=H_{t} \mathbb{E}_{t \mid \Phi_{s]}} \otimes I_{\mid \Phi_{[s}}$ for all $\left.\left.s \in\right] t,+\infty\right]$.

Lemma 8.3. The family $\left(H_{s}^{\circ}\right)_{s \geqq 0}$ is an Hilbert-Schmidt martingale.

Proof. Let $\left(u^{n}\right)_{n \in \mathbb{N}}$ be an o.n.b. of $\Phi_{t]}$ and $\left(v^{n}\right)_{n \in \mathbb{N}}$ be an o.n.b. of $\Phi_{t]}^{\perp}$. The family $\left(w^{n}\right)_{n \in \mathbb{N}}$, defined by $w^{2 n}=u^{n}, w^{2 n+1}=v^{n}$ is then an o.n.b. of $\Phi$. One has

$$
\begin{aligned}
\sum_{n=0}^{\infty}\left\|H_{\infty}^{\circ} w^{n}\right\|^{2} & =\sum_{n=0}^{\infty}\left\|H_{\infty}^{\circ} u^{n}\right\|^{2}+\sum_{n=0}^{\infty}\left\|H_{\infty}^{\circ} v^{n}\right\|^{2} \\
& =\sum_{n=0}^{\infty}\left\|H_{t} \mathbb{E}_{t} u^{n}\right\|^{2}+\sum_{n=0}^{\infty}\left\|H_{t} \mathbb{E}_{t} v^{n}\right\|^{2} \\
& =\sum_{n=0}^{\infty}\left\|H_{t} u^{n}\right\|^{2}=\left\|H_{t}\right\|_{H S}^{2}<\infty .
\end{aligned}
$$

So $H_{\infty}^{\circ}$ is an Hilbert-Schmidt operator on $\Phi$. 
The martingale $\left(H_{\infty, s}^{\circ}\right)_{s \geqq 0}$ associated to $H_{\infty}^{\circ}$ is an Hilbert-Schmidt martingale and is defined by

$$
\begin{aligned}
H_{\infty, s}^{\circ} & =\mathbb{E}_{s} H_{\infty \mid \Phi_{s]}}^{\circ} \otimes I_{\mid \Phi_{s]}}=\mathbb{E}_{s} H_{t} \mathbb{E}_{t \mid \Phi_{s]}} \otimes I_{\mid \Phi_{s]}} \\
& = \begin{cases}H_{t} \mathbb{E}_{t \mid \Phi_{s]}} \otimes I_{\mid \Phi_{s]}} & \text { si } s>t \\
\mathbb{E}_{s} H_{t \mid \Phi_{s]}} \otimes I_{\mid \Phi_{s]}} & \text { si } s \leqq t\end{cases} \\
& =H_{s}^{\circ} .
\end{aligned}
$$

So $\left(H_{s}^{\circ}\right)_{s \geqq 0}$ is an Hilbert-Schmidt martingale on $\overline{\mathbb{R}^{+}}$and the lemma is proved.

By this lemma one can apply the results of the first case. One obtains the required integral representation on $\Phi$, along with the appropriate estimates for the norm of the coefficients.

Proposition 8.2 shows that any Hilbert-Schmidt operator from $\Phi_{t]}$ into $\Phi_{t]}$ admits a non-commutative stochastic integral representation such that all the coefficients are Hilbert-Schmidt operators on the corresponding space $\Phi_{s]}$. The same kind of conditions as for the iteration scheme of the previsible representation of Brownian functionals are verified here. We then can iterate this integral representation. Let us precise a "short notation."

Let $E_{n}=\{+, \circ,-\}^{n}, E_{n}^{*}=\{+,-\}^{n}$. For an element $E=\left(\varepsilon_{1}, \ldots, \varepsilon_{n}\right)$ of $E_{n}$ we denote $n_{\circ}(E)$ the number of elements $\varepsilon_{i}$ which are equal to $\circ$.

In the following we use families of operators $H_{t_{n} \cdots t_{1}}^{\varepsilon_{n} \cdots \varepsilon_{1}}$, indexed by $E_{n} \times \mathscr{P}_{n}$ or $E_{n}^{*} \times \mathscr{P}_{n}$. We denote

$$
\sum_{E \in E_{n}} \int_{\mathscr{P}_{n}} H_{M}^{E} d A_{M}^{E}=\sum_{\varepsilon_{1}, \ldots, \varepsilon_{n} \in\{+, 0,-\}} \int_{0}^{\infty} \int_{0}^{t_{n}} \cdots \int_{0}^{t_{2}} H_{t_{n} \cdots t_{1}}^{\varepsilon_{n} \cdots \varepsilon_{1}} d A_{t_{1}}^{\varepsilon_{1}} \cdots d A_{t_{n}}^{\varepsilon_{n}} .
$$

Remark that, in the notations $H_{M}^{E}$ or $d A_{M}^{E}$, the elements of $E$ and of $M$ are not taken in the same order.

Proposition 8.4. Let $H$ be an Hilbert-Schmidt operator from $\Phi$ into $\Phi$. For all $N \in \mathbb{N}^{*}, H$ admits, on all $\Phi$, an integral representation of the form

$$
H=\sum_{n=0}^{N-1} \sum_{E \in E_{n}} \int_{\mathscr{P}_{n}}(-1)^{n_{\circ}(E)} \mathbb{E}\left[H_{M}^{E} \mathbb{1}\right] I d A_{M}^{E}+\sum_{E \in E_{N}} \int_{\mathscr{P}_{N}}(-1)^{n_{\circ}(E)} H_{M}^{E} d A_{M}^{E} .
$$

Furthermore, for all $M=\left(t_{1}<\cdots<t_{N}\right) \in \mathscr{P}_{N}$, all $E \in E_{N}$, the operator $H_{M}^{E}$ is an Hilbert-Schmidt operator from $\Phi_{\left.t_{1}\right]}$ into $\Phi_{\left.t_{1}\right]}$; and one has the following estimates: for all $E \in E_{N}^{*}$,

$$
\int_{\mathscr{P}_{N}}\left\|H_{M}^{E}\right\|_{H S}^{2} d M \leqq\|H\|_{H S}^{2},
$$

for all $E \in E_{N}$, all $0<T<\infty$,

$$
\int_{\mathscr{P}_{N} \cap[0, T]^{N}}\left\|H_{M}^{E}\right\|_{H S}^{2} d M \leqq T^{N}\|H\|_{H S}^{2}
$$

Proof. By Proposition 8.2, Proposition 8.4 is verified for $N=1$. Working by induction on $N$, suppose that the proposition is true for every $k \leqq N$. 
For all $M \in \mathscr{P}_{N}, E \in E_{N}$, the operator $H_{M}^{E}$ is Hilbert-Schmidt from $\Phi_{t_{1}}$ into $\Phi_{\left.t_{1}\right]}$, so by Proposition 8.2 , it admits, on all $\Phi$, an integral representation of the form

$$
\begin{aligned}
H_{M}^{E} & =\mathbb{E}\left[H_{M}^{E} \mathbb{1}\right] I-\int_{0}^{t_{1}}\left(H_{M}^{E}\right)_{s}^{\circ} d A_{s}^{\circ}+\int_{0}^{t_{1}}\left(H_{M}^{E}\right)_{s}^{-} d A_{s}^{-}+\int_{0}^{t_{1}}\left(H_{M}^{E}\right)_{s}^{+} d A_{s}^{+} \\
& =\mathbb{E}\left[H_{M}^{E} \mathbb{1}\right] I-\int_{0}^{t_{1}} H_{M \cup(s)}^{E \cup(\circ)} d A_{s}^{\circ}+\int_{0}^{t_{1}} H_{M \cup(s)}^{E \cup(-)} d A_{s}^{-}+\int_{0}^{t_{1}} H_{M \cup(s)}^{E \cup(+)} d A_{s}^{+} .
\end{aligned}
$$

Reintroducing this representation in that of $H$, one gets

$$
\begin{aligned}
H= & \sum_{n=0}^{N-1} \sum_{E \in E_{n}} \int_{\mathscr{P}_{n}}(-1)^{n_{\circ}(E)} \mathbb{E}\left[H_{M}^{E} \mathbb{1}\right] I d A_{M}^{E}+\sum_{E \in E_{N}} \int_{\mathscr{P}_{N}}(-1)^{n_{\circ}(E)} \mathbb{E}\left[H_{M}^{E} \mathbb{1}\right] I d A_{M}^{E} \\
& +\sum_{E \in E_{N}} \sum_{\varepsilon \in\{+, \circ,-\}} \int_{\mathscr{P}_{N}} \int_{0}^{t_{1}}(-1)^{n_{\circ}(E)}(-1)^{n_{\circ}(\varepsilon)}\left(H_{M}^{E}\right)_{s}^{\varepsilon} d A_{S}^{\varepsilon} d A_{M}^{E} \\
= & \sum_{n=0}^{N} \sum_{E \in E_{n}} \int_{\mathscr{P}_{n}}(-1)^{n_{\circ}(E)} \mathbb{E}\left[H_{M}^{E} \mathbb{1}\right] I d A_{M}^{E}+\sum_{E \in E_{N+1}} \int_{\mathscr{P}_{N+1}}(-1)^{n_{\circ}(E)} H_{M}^{E} d A_{M}^{E} .
\end{aligned}
$$

This proves the required integral representation at level $N+1$.

Let us prove that the other conditions are also satisfied. For all $M \in \mathscr{P}_{N}, E \in E_{N}$, let $\alpha_{M}^{E}(s)=\left\|\left(H_{M}^{E}\right)_{s \mid \Phi_{s]}}^{\circ}\right\|_{H S}^{2}, s \leqq t_{1}$. One knows, by the induction hypothesis, that the operators $\left(H_{M}^{E}\right)_{s}^{ \pm}$are Hilbert-Schmidt from $\Phi_{s]}$ into $\Phi_{s]}$ and that

$$
\left\|\left(H_{M}^{E}\right)_{\left.s \mid \Phi_{s}\right]}^{ \pm}\right\|_{H S}^{2} \leqq \alpha_{M}^{\prime E}(s)
$$

where $\alpha_{M}^{\prime} E$ is the absolutely continuous part of the Stieltjes measure associated to $\alpha_{M}^{E}$ (which is a non-decreasing function on $\left[0, t_{1}\right]$ ). So one has

$$
\begin{aligned}
\int_{\mathscr{P}_{N}} \int_{0}^{t_{1}}\left\|\left(H_{M}^{E}\right)_{s \mid \Phi_{s]}}^{ \pm}\right\|_{H S}^{2} d s d M & \leqq \int_{\mathscr{P}_{N}}\left(\alpha_{M}^{E}\left(t_{1}\right)-\alpha_{M}^{E}(0)\right) d M \\
& \leqq \int_{\mathscr{P}_{N}}\left\|H_{M}^{E}\right\|_{H S}^{2} d M
\end{aligned}
$$

and

$$
\begin{aligned}
\int_{\mathscr{P}_{N} \cap[0, T]^{N}} \int_{0}^{t_{1}}\left\|\left(H_{M}^{E}\right)_{s \mid \Phi_{s]}}^{\circ}\right\|_{H S}^{2} d s d M & \leqq \int_{\mathscr{P}_{N} \cap[0, T]^{N}}\left\|H_{M}^{E}\right\|_{H S}^{2} \int_{0}^{t_{1}} d s d M \\
& \leqq T \int_{\mathscr{P}_{N} \cap[0, T]^{N}}\left\|H_{M}^{E}\right\|_{H S}^{2} d M .
\end{aligned}
$$

The conclusion follows by induction.

We have proved that one can iterate $N$ times the non-commutative stochastic integral representation of Hilbert-Schmidt operators on Fock space. We are going to prove that this iteration converges when $N$ tends to $+\infty$. 


\section{Convergence of the Iteration}

Let $g \in L_{l b}^{2}\left(\mathbb{R}^{+}\right)$, let $\varepsilon \in\{+, \circ,-\}$, we denote

$$
\begin{aligned}
& g^{\varepsilon}= \begin{cases}g & \text { if } \varepsilon=0 \text { or }-, \\
\mathbb{1} & \text { if } \varepsilon=+,\end{cases} \\
& g_{\varepsilon}= \begin{cases}g & \text { if } \varepsilon=0 \text { or }+, \\
\mathbb{1} & \text { if } \varepsilon=-\end{cases}
\end{aligned}
$$

We also denote $M_{g}(t)=\max \left(1, \sup _{s \in[0, t]}|g(s)|\right)$.

For $E=\left(\varepsilon_{1}, \ldots, \varepsilon_{n}\right) \in E_{n}$ and $M=\left(t_{1}, \ldots, t_{n}\right) \in \mathscr{P}_{n}$, denote

$$
g^{E}(M)=\prod_{i=1}^{n} g^{\varepsilon_{l}}\left(t_{i}\right) \quad \text { and } \quad g_{E}(M)=\prod_{i=1}^{n} g_{\varepsilon_{i}}\left(t_{i}\right) .
$$

Finally, let $\mathscr{E}_{l b}^{c}$ be the space of finite linear combinations of elements $\varepsilon(u)$, where $u$ is an element of $L_{l b}^{2}\left(\mathbb{R}^{+}\right)$with compact support.

Proposition 9.1. Let $H$ be an Hilbert-Schmidt operator from $\Phi$ into $\Phi$. Let $N \in \mathbb{N}^{*}$, let

$$
R_{N}=\sum_{E \in E_{N}} \int_{\mathscr{P}_{N}}(-1)^{n_{\circ}(E)} H_{M}^{E} d A_{M}^{E}
$$

be the remainder of the integral representation (iterated $N$ times) of $H$ given by Proposition 8.4. Then, for all $f, g \in \mathscr{E}_{l b}^{c}$, the term $\left\langle g, R_{N} f\right\rangle$ converges to 0 when $N$ tends to $+\infty$.

So, in the sense of this weak convergence, one has

$$
H=\sum_{n=0}^{\infty} \sum_{E \in E_{n}} \int_{\mathscr{P}_{n}} \mathbb{E}\left[H_{M}^{E} \mathbb{1}\right] I d A_{M}^{E} \text {. }
$$

Proof. Let $\varepsilon(f)$ and $\varepsilon(g)$ be two elements of $\mathscr{E}_{l b}^{c}$. Let $T \in \mathbb{R}^{+}$be such that the supports of $f$ and $g$ are included in $[0, T]$. One has, by (3),

$$
\begin{aligned}
\left|\left\langle\varepsilon(g), R_{N} \varepsilon(f)\right\rangle\right|= & \left|\sum_{E \in E_{N}} \int_{\mathscr{P}_{N}}(-1)^{n_{\circ}(E)} \bar{g}_{E}(M) f^{E}(M)\left\langle\varepsilon(g), H_{M}^{E} \varepsilon(f)\right\rangle d M\right| \\
\leqq & \sum_{E \in E_{N}} \int_{\mathscr{P}_{N}}\left|g_{E}(M)\right|\left|f^{E}(M)\right|\left|\left\langle\varepsilon(g), H_{M}^{E} \varepsilon(f)\right\rangle\right| d M \\
\leqq & \sum_{E \in E_{N}}\left(\int_{\mathscr{P}_{N} \cap[0, T]^{N}}\left|g_{E}(M)\right|^{2}\left|f^{E}(M)\right|^{2} d M\right)^{1 / 2} \\
& \times\left(\int_{\mathscr{P}_{N} \cap[0, T]^{N}}\left|\left\langle\varepsilon(g), H_{M}^{E} \varepsilon(f)\right\rangle\right|^{2} d M\right)^{1 / 2} \\
& \leqq \sum_{E \in E_{N}}\left(\int_{\mathscr{P}_{N} \cap[0, T]^{N}}\left|g_{E}(M)\right|^{2}\left|f^{E}(M)\right|^{2} d M\right)^{1 / 2} \\
& \times\left(\int_{\mathscr{P}_{N} \cap[0, T]^{N}}\|\varepsilon(g)\|^{2}\left\|H_{M}^{E}\right\|^{2}\|\varepsilon(f)\|^{2} d M\right)^{1 / 2}
\end{aligned}
$$




$$
\begin{aligned}
\leqq & \sum_{E \in E_{N}}\left(M_{g}(t) M_{f}(t)\right)^{N}\left(\int_{\mathscr{P}_{N} \cap[0, T]^{N}} d M\right)^{1 / 2}\|\varepsilon(g)\|\|\varepsilon(f)\| \\
& \times\left(\int_{\mathscr{P}_{N} \cap[0, T]^{N}}\left\|H_{M}^{E}\right\|_{H S}^{2} d M\right)^{1 / 2} \\
\leqq & 3^{N}\left(M_{g}(t) M_{f}(t)\right)^{N} \frac{T^{N / 2}}{\sqrt{N !}}\|\varepsilon(g)\|\|\varepsilon(f)\| T^{N}\|H\|_{H S} .
\end{aligned}
$$

This last term converges to 0 when $N$ tends to $+\infty$.

\section{Computation of the Kernel from the Iteration}

We begin here the most important and the most technical part of this work. We have seen from Proposition 9.1 that an Hilbert-Schmidt operator is, in some way, the sum of a series of iterated non-commutative stochastic integrals of scalar operators. We now prove that one can reconstruct the Maassen-Meyer kernel of the operator from this iteration and describe it completely.

Let $f$ be an element of $\Phi$. We denote, for all $t \in \mathbb{R}^{+},\left(f_{t}^{+}\right)_{t \geqq 0}$ the martingale associated with $f$ and $\left(f_{t}^{-}\right)_{t \geqq 0}$ the previsible process which appears in its previsible representation. Let $d W_{t}^{+}=d W_{t}$ and $d W_{t}^{-}=d t$.

For $E=\left(\varepsilon_{1}, \ldots, \varepsilon_{n}\right) \in E_{n}^{*}$, and $M=\left(t_{1}<\cdots<t_{n}\right) \in \mathscr{P}_{n}$, put

$$
f_{M}^{E}=f_{t_{n} \cdots t_{1}}^{\varepsilon_{n} \cdots \varepsilon_{1}}=\left(\cdots\left(\left(f_{t_{n}}^{\varepsilon_{n}}\right)_{t_{n-1}}^{\varepsilon_{n-1}}\right) \cdots\right)_{t_{1}}^{\varepsilon_{1}} .
$$

We also denote $d W_{M}^{E}=d W_{t_{1}}^{\varepsilon_{1}} \cdots d W_{t_{n}}^{\varepsilon_{n}}$.

Proposition 10.1. Let $H$ be an Hilbert-Schmidt operator from $\Phi$ into $\Phi$, let $f \in \Phi$. One has, for all $N \in \mathbb{N}^{*}$,

$$
H f=\sum_{n=0}^{N-1} \sum_{E \in E_{n}^{*}} \int_{\mathscr{P}_{n}} \mathbb{E}\left[H_{M}^{E} \mathbb{1}\right] \mathbb{E}\left[f_{M}^{E}\right] d W_{M}^{E}+\sum_{E \in E_{N}^{*}} \int_{\mathscr{P}_{N}} H_{M}^{E} f_{M}^{E} d W_{M}^{E},
$$

where the operators $H_{M}^{E}$ are those given by Proposition 8.4, with $E \in \bigcup_{n} E_{n}^{*}$.

Proof. From Proposition 8.2 and identity (4), defining the extensions of the noncommutative stochastic integrals, one has, for every operator $H_{t}$ which is HilbertSchmidt from $\Phi_{t]}$ into $\Phi_{t]}$, every $f_{t} \in \Phi_{t]}$,

$$
\begin{aligned}
H_{t} f_{t}= & \mathbb{E}\left[H_{t} \mathbb{1}\right] \mathbb{E}\left[f_{t}\right]+\int_{0}^{t} H_{s}^{\circ} f_{s}^{-} d W_{s}^{+}-\int_{0}^{t} H_{s}^{\circ} f_{s}^{-} d W_{s}^{+} \\
& +\int_{0}^{t} H_{s}^{-} f_{s}^{-} d W_{s}^{-}+\int_{0}^{t} H_{s}^{+} f_{s}^{+} d W_{s}^{+} \\
H_{t} f_{t}= & \mathbb{E}\left[H_{t} \mathbb{1}\right] \mathbb{E}\left[f_{t}\right]+\int_{0}^{t} H_{s}^{-} f_{s}^{-} d W_{s}^{-}+\int_{0}^{t} H_{s}^{+} f_{s}^{+} d W_{s}^{+} .
\end{aligned}
$$


So the proposition is verified for $N=1$. Suppose that the proposition is verified for all ranks up to $N$. One then has

$$
H f=\sum_{n=0}^{N-1} \sum_{E \in E_{n}^{*}} \int_{\mathscr{P}_{n}} \mathbb{E}\left[H_{M}^{E} \mathbb{1}\right] \mathbb{E}\left[f_{M}^{E}\right] d W_{M}^{E}+\sum_{E \in E_{N}^{*}} \int_{\mathscr{P}_{N}} H_{M}^{E} f_{M}^{E} d W_{M}^{E} .
$$

The operators $H_{M}^{E}, M \in \mathscr{P}_{N}, E \in E_{N}^{*}$, are Hilbert-Schmidt operators from $\Phi_{\left.t_{1}\right]}$ into $\Phi_{\left.t_{1}\right]}$ (where $t_{1}$ is the smallest element of $M$ ). In this case, $f_{M}^{E}$ is an element of $\Phi_{t_{1}}$, applying (6), one has

$$
H_{M}^{E} f_{M}^{E}=\mathbb{E}\left[H_{M}^{E} \mathbb{1}\right] \mathbb{E}\left[f_{M}^{E}\right]+\int_{0}^{t_{1}}\left(H_{M}^{E}\right)_{s}^{-} f_{s}^{-} d W_{s}^{-}+\int_{0}^{t}\left(H_{M}^{E}\right)_{s}^{+} f_{s}^{+} d W_{s}^{+} .
$$

Introducing this equality in the representation of $H f$, one obtains

$$
\begin{aligned}
H f= & \sum_{n=0}^{N-1} \sum_{E \in E_{n}^{*}} \int_{\mathscr{P}_{n}} \mathbb{E}\left[H_{M}^{E} \mathbb{1}\right] \mathbb{E}\left[f_{M}^{E}\right] d W_{M}^{E}+\sum_{E \in E_{N}^{*}} \int_{\mathscr{P}_{N}} \mathbb{E}\left[H_{M}^{E} \mathbb{1}\right] \mathbb{E}\left[f_{M}^{E}\right] d W_{M}^{E} \\
& +\sum_{E \in E_{N}^{*}} \sum_{\varepsilon \in\{+,-\}} \int_{\mathscr{P}_{N}} \int_{0}^{t_{1}} H_{M \cup(s)}^{E \cup(\varepsilon)} f_{M \cup(s)}^{E \cup(\varepsilon)} d W_{s}^{\varepsilon} d W_{M}^{E} \\
= & \sum_{n=0}^{N} \sum_{E \in E_{n}^{*}} \int_{\mathscr{P}_{n}} \mathbb{E}\left[H_{M}^{E} \mathbb{1}\right] \mathbb{E}\left[f_{M}^{E}\right] d W_{M}^{E}+\sum_{E \in E_{N+1}} \int_{\mathscr{P}_{N+1}} H_{M}^{E} f_{M}^{E} d W_{M}^{E},
\end{aligned}
$$

which is the required representation at rank $N+1$.

This proposition gives a representation of $H f$ in two terms: the first one must be the begining of the chaotic expansion of $H f$, the second one must converge to 0 when $N$ tends to $+\infty$.

Let $\Xi$ be the set of $f \in \Phi$ such that

$$
\int_{\mathscr{P}} 4^{|A|} \widehat{f}(A)^{2} d A<\infty .
$$

It is a dense subspace of $\Phi$.

Proposition 10.2. Let $H$ be an Hilbert-Schmidt operator from $\Phi$ into $\Phi$, let $N \in$ $\mathbb{N}$, let $f \in \Phi$ and let

$$
r_{N}(f)=\sum_{E \in E_{N}^{*}} \int_{\mathscr{P}_{N}} H_{M}^{E} f_{M}^{E} d W_{M}^{E}
$$

be the remainder appearing in the representation of $H f$ given by Proposition 10.1 . Let $m \in \mathbb{N}$ be fixed, let $g_{m}$ be an element of $L^{2}\left(\Sigma_{m}\right)$ and $I_{m}\left(g_{m}\right)$ be the element of the $m^{\text {th }}$ associated chaos.

Then, for all $f \in \Xi$, the term $\left\langle I_{m}\left(g_{m}\right), r_{N}(f)\right\rangle$ converges to 0 when $N$ tends to $+\infty$.

Proof. Let $f \in \Phi$, one has

$$
\left|\left\langle I_{m}\left(g_{m}\right), r_{N}(f)\right\rangle\right|=\left|\sum_{E \in E_{N}^{*}}\left\langle\int_{\mathscr{P}_{m}} g_{m}(M) d W_{M}, \int_{\mathscr{P}_{N}} H_{M}^{E} f_{M}^{E} d W_{M}^{E}\right\rangle\right| .
$$

For $E=\left\{\varepsilon_{1}, \ldots, \varepsilon_{N}\right\} \in E_{N}^{*}$, let $n_{+}(E)$ be the number of elements of $E$ which are equal to + . Suppose that $n_{+}(E)=n \leqq N$. The term $\int_{\mathscr{P}_{N}} H_{M}^{E} f_{M}^{E} d W_{M}^{E}$ is an iterated 
integral with respect to Brownian motion and to time. One knows that there are exactly $n$ integrations with respect to Brownian motion and $N-n$ integrations with respect to time. Let $I_{+}(E)$ be the subset of $\{1, \ldots, N\}$ constituted by the indices $k$ such that $\varepsilon_{k}=+$.

We are going to reorder the integral $\int_{\mathscr{P}_{N}} H_{M}^{E} f_{M}^{E} d W_{M}^{E}$ so as to first integrate with respect to the $N-n$ terms in $d t$, then with respect to the $n$ terms in $d W_{t}$. This gives, if $I_{+}(E)=\left\{i_{1}<\cdots<i_{n}\right\}$,

$$
\begin{array}{r}
\int_{0}^{\infty} \int_{0}^{t_{t_{n}}} \cdots \int_{0}^{t_{i_{2}}}\left[\int_{t_{l_{n}}}^{\infty} \int_{t_{l_{n}}}^{t_{N}} \cdots \int_{t_{t_{n}}}^{t_{l_{n}+2}} \cdots \int_{t_{l_{k}}}^{t_{l_{k}}+1} \cdots \int_{t_{l_{k}}}^{t_{t_{k}+2}} \cdots \int_{0}^{t_{l_{1}}} \cdots \int_{0}^{t_{1}} T_{t_{N} \cdots t_{1}}^{\varepsilon_{N} \cdots \varepsilon_{1}} f_{t_{N} \cdots t_{1}}^{\varepsilon_{N} \cdots \varepsilon_{1}}\right. \\
\left.d t_{1} \cdots d t_{i_{1}-1} d t_{i_{1}+1} \cdots d t_{i_{k}-1} d t_{i_{k}+1} \cdots d t_{N}\right] d W_{t_{l_{1}}} \cdots d W_{t_{l_{n}}}
\end{array}
$$

In this way, one integrates with respect to Brownian motion along $\Sigma_{n}$ and with respect to time along a subset of $\Sigma_{N-n}$ denoted $S_{N, n}\left(I_{+}(E)\right.$ ) (indeed this reordering is entirely determined by the integer $N$ and the subset $I_{+}(E)$ of $\{1, \ldots, N\}$ indicating which indices are moved in front). In this way

$$
\begin{aligned}
& \left|\left\langle I_{m}\left(g_{m}\right), r_{N}(f)\right\rangle\right| \\
& =\left|\sum_{n=0}^{N} \sum_{\substack{E \in E_{N}^{*} \\
n_{+}(E)=n}}\left\langle\int_{\mathscr{P}_{m}} g_{m}(M) d W_{M}, \int_{M^{\prime} \in \mathscr{P}_{n}}\left[\int_{M \in S_{N, n\left(I_{+}(E)\right)}} H_{M \cup M^{\prime}}^{E} f_{M \cup M^{\prime}}^{E} d M\right] d W_{M^{\prime}}\right\rangle\right| \\
& =\mid \sum_{n=0}^{m} \sum_{\substack{E \in E_{N}^{*} \\
n_{+}(E)=n}} \int_{M^{\prime} \in \mathscr{P}_{n}}\left\langle\int_{M^{\prime \prime} \in \mathscr{P}_{m-n}} g_{m}\left(M^{\prime \prime} \cup M^{\prime}\right) d W_{M^{\prime \prime}}\right. \\
& \left.\int_{M \in S_{N, n}\left(I_{+}(E)\right)} H_{M \cup M^{\prime}}^{E} f_{M \cup M^{\prime}}^{E} d M\right\rangle d M^{\prime} \\
& =\mid \sum_{n=0}^{m} \sum_{\substack{E \in E_{N}^{*} \\
n_{+}(E)=n}} \int_{M^{\prime} \in \mathscr{P}_{n}} \int_{M \in S_{N, n}\left(I_{+}(E)\right)}\left\langle\int_{M^{\prime \prime} \in \mathscr{P}_{m-n}} g_{m}\left(M^{\prime \prime} \cup M^{\prime}\right) d W_{M^{\prime \prime}},\right. \\
& \left.H_{M \cup M^{\prime}}^{E} f_{M \cup M^{\prime}}^{E}\right\rangle d M d M^{\prime} \\
& \leqq \sum_{n=0}^{m} \sum_{\substack{E \in E_{N}^{*} \\
n_{+}(E)=n}} \int_{M^{\prime} \in \mathscr{P}_{n}} \int_{M \in S_{N, n}\left(I_{+}(E)\right)}\left\|\int_{M^{\prime \prime} \in \mathscr{P}_{m-n}} g_{m}\left(M^{\prime \prime} \cup M^{\prime}\right) d W_{M^{\prime \prime}}\right\| \\
& \times\left\|H_{M \cup M^{\prime}}^{E}\right\|\left\|f_{M \cup M^{\prime}}^{E}\right\| d M d M^{\prime}
\end{aligned}
$$




$$
\begin{aligned}
\leqq & \sum_{n=0}^{m} \sum_{E \in E_{N}^{*}}\left(\int_{n_{+}(E)=n} \int_{M^{\prime} \in \mathscr{P}_{n}}\left\|\int_{M \in S_{N, n}\left(I_{+}(E)\right)} \int_{M^{\prime \prime} \in \mathscr{P}_{m-n}} g_{m}\left(M^{\prime \prime} \cup M^{\prime}\right) d W_{M^{\prime \prime}}\right\|^{2}\right. \\
& \left.\times\left\|f_{M \cup M^{\prime}}^{E}\right\|^{2} d M d M^{\prime}\right)^{1 / 2}\left(\int_{M^{\prime} \in \mathscr{P}_{n}} \int_{M \in S_{N, n}\left(I_{+}(E)\right)}\left\|H_{M \cup M^{\prime}}^{E}\right\|^{2} d M d M^{\prime}\right)^{1 / 2} \\
\leqq & \sum_{n=0}^{m} \sum_{\sum_{n_{+}(E)=n}}\left(\int_{M^{\prime} \in \mathscr{P}_{n}}\left(\int_{M^{\prime \prime} \in \mathscr{P}_{m-n}} g_{m}^{2}\left(M^{\prime \prime} \cup M^{\prime}\right) d M^{\prime \prime}\right)\right. \\
& \left.\times\left(\int_{M \in S_{N, n}\left(I_{+}(E)\right)}\left\|f_{M \cup M^{\prime}}^{E}\right\|^{2} d M\right) d M^{\prime}\right)^{1 / 2}\left(\int_{\mathscr{P}_{N}}\left\|H_{M}^{E}\right\|^{2} d M\right)^{1 / 2}
\end{aligned}
$$

But one must remark that, for all $s \leqq t$, all $f \in \Phi$, one has $f_{t s}^{+-}=f_{s}^{-}$. So, one has $\left\|f_{M \cup M^{\prime}}^{E}\right\|^{2}=\left\|f_{M}^{\{-\}^{n}}\right\|^{2}$. This gives

$$
\left|\left\langle I_{m}\left(g_{m}\right), r_{N}(f)\right\rangle\right| \leqq \sum_{n=0}^{m} \sum_{\substack{E \in E_{N}^{*} \\ n_{+}(E)=n}}\left\|I_{m}\left(g_{m}\right)\right\|\left(\int_{\Sigma_{N-n}}\left\|f_{M}^{\{-\}^{n}}\right\|^{2} d M\right)^{1 / 2}\|H\|_{H S} .
$$

For $f \in \Phi, i \in \mathbb{N}$, we denote by $f_{[l}$ the vector

$$
\int_{\substack{A \in \mathscr{P} \\|A| \geqq i}} \widehat{f}(A) d W_{A}
$$

that is $f$ minus its projection on the first $i$ chaos (including the chaos of order $0: \mathbb{E}[f])$. One has

$$
\begin{aligned}
\left|\left\langle I_{m}\left(g_{m}\right), r_{N}(f)\right\rangle\right| & \leqq\left\|I_{m}\left(g_{m}\right)\right\|\|H\|_{H S} \sum_{n=0}^{m} \sum_{\substack{E \in E_{N}^{*} \\
n_{+}(E)=n}}\left\|f_{[N-n}\right\| \\
& \leqq\left\|I_{m}\left(g_{m}\right)\right\|\|H\|_{H S} 2^{N}\left\|f_{[N-m}\right\| .
\end{aligned}
$$

But, one has

$$
\begin{aligned}
2^{N-m}\left\|f_{[N-m}\right\| & =2^{N-m}\left(\int_{\substack{A \in \mathscr{P} \\
|A| \geqq N-m}} \widehat{f}(A)^{2} d A\right)^{1 / 2}=\left(\int_{\substack{A \in \mathscr{P} \\
|A| \geqq N-m}} 4^{N-m} \widehat{f}(A)^{2} d A\right)^{1 / 2} \\
& \leqq\left(\int_{\substack{A \in \mathscr{P} \\
|A| \geqq N-m}} 4^{|A|} \widehat{f}(A)^{2} d A\right)^{1 / 2} \cdot
\end{aligned}
$$

If $f$ is an element of $\Xi$, this last term converges to 0 when $N$ tends to $+\infty$. So one concludes.

This result of convergence allows to express the chaotic decomposition of $H f$ in terms of the decomposition of $f$. 
In the following we denote by $\widetilde{\Xi}$ the space of Maassen test-vectors presented in Sect. 6 .

Proposition 10.3. Let $H$ be an Hilbert-Schmidt operator from $\Phi$ into $\Phi$, let $f \in \Phi$ and $m \in \mathbb{N}$. If one denotes by $I_{m}(H f)$ the projection of $H f$ on the $m^{\text {th }}$ chaos, then

$$
I_{m}(H f)=\sum_{n=m}^{\infty} \sum_{\substack{E \in E_{n}^{*} \\ n_{+}(E)=m}} \int_{\mathscr{P}_{n}} \mathbb{E}\left[H_{M}^{E} \mathbb{1}\right] \mathbb{E}\left[f_{M}^{E}\right] d W_{M}^{E}
$$

where the convergence of the series is weak if $f \in \Xi$ and strong if $f \in \widetilde{\Xi}$.

Proof. It is clear that the projection on the $m^{\text {th }}$ chaos of

$$
\sum_{n=0}^{N} \sum_{E \in E_{n}^{*}} \int_{\mathscr{P}_{n}} \mathbb{E}\left[H_{M}^{E} \mathbb{1}\right] \mathbb{E}\left[f_{M}^{E}\right] d W_{M}^{E}
$$

is equal to

$$
\sum_{n=m}^{N} \sum_{\substack{E \in E_{n}^{*} \\ n_{+}(E)=m}} \int_{\mathscr{P}_{n}} \mathbb{E}\left[H_{M}^{E} \mathbb{1}\right] \mathbb{E}\left[f_{M}^{E}\right] d W_{M}^{E} .
$$

So, by Propositions 10.1 and 10.2 , for all $f \in \Xi, I_{m}(H f)$ is the weak limit of the expression (7) when $N$ tends to $+\infty$.

In order to show that this limit occurs in the strong sense on $\widetilde{\Xi}$, it is sufficient to prove that, for all $f \in \widetilde{\Xi}$, the set of terms given by (7) is bounded in norm, uniformly in $N$. One has

$$
\begin{aligned}
& \sum_{n=m}^{N} \sum_{\substack{E \in E_{n}^{*} \\
n_{+}(E)=m}}\left\|\int_{\mathscr{P}_{n}} \mathbb{E}\left[H_{M}^{E} \mathbb{1}\right] \mathbb{E}\left[f_{M}^{E}\right] d W_{M}^{E}\right\| \\
& =\sum_{n=m}^{N} \sum_{\substack{E \in E_{n}^{*} \\
n_{+}(E)=m}}\left\|\int_{M \in \mathscr{P}_{m}} \int_{M^{\prime} \in S_{n, m}\left(I_{+}(E)\right)} \mathbb{E}\left[H_{M \cup M^{\prime}}^{E} \mathbb{1}\right] \mathbb{E}\left[f_{M \cup M^{\prime}}^{E}\right] d M^{\prime} d W_{M}\right\| \\
& =\sum_{n=m}^{N} \sum_{\substack{E \in E_{n}^{*} \\
n_{+}(E)=m}}\left(\int_{M \in \mathscr{P}_{m}}\left(\int_{M^{\prime} \in S_{n, m}\left(I_{+}(E)\right)} \mathbb{E}\left[H_{M \cup M^{\prime}}^{E} \mathbb{1}\right] \mathbb{E}\left[F_{M \cup M^{\prime}}^{E}\right] d M^{\prime}\right)^{2} d M\right)^{1 / 2} \\
& \leqq \sum_{n=m}^{N} \sum_{\substack{E \in E_{n}^{*} \\
n_{+}(E)=m}}\left(\int_{M \in \mathscr{P}_{m}}\left(\int_{M^{\prime} \in S_{n, m}\left(I_{+}(E)\right)} \mathbb{E}\left[H_{M \cup M^{\prime}}^{E} \mathbb{1}\right]^{2} d M^{\prime}\right)\right. \\
& \left.\times\left(\int_{M^{\prime} \in S_{n, m}\left(I_{+}(E)\right)} \mathbb{E}\left[f_{M \cup M^{\prime}}^{E}\right]^{2} d M^{\prime}\right) d M\right)^{1 / 2} \\
& \leqq \sum_{n=m}^{N} \sum_{\substack{E \in E_{n}^{*} \\
n_{+}(E)=m}}\left(\int_{M \in \mathscr{P}_{m}}\left(\int_{M^{\prime} \in S_{n, m}\left(I_{+}(E)\right)}\left\|H_{M \cup M^{\prime}}^{E}\right\|^{2} d M^{\prime}\right) d M\right)^{1 / 2}\left\|I_{n-m}(f)\right\|
\end{aligned}
$$




$$
\begin{aligned}
& \leqq \sum_{n=m}^{N} \sum_{\substack{E \in E_{n}^{*} \\
n_{+}(E)=m}}\|H\|_{H S}\left\|I_{n-m}(f)\right\| \leqq\|H\|_{H S}-\sum_{n=m}^{N} 2^{n}\left\|I_{n-m}(f)\right\| \\
& \leqq\|H\|_{H S} 2^{m} \sum_{n=0}^{N} 2^{n}\left\|I_{n}(f)\right\| .
\end{aligned}
$$

But, if $f$ is an element of $\widetilde{\Xi}$, one has

$$
\left\|I_{n}(f)\right\|=\left(\int_{\mathscr{P}_{n}} \widehat{f}(A)^{2} d A\right)^{1 / 2} \leqq\left(\int_{\mathscr{P}_{n} \cap[0, T]^{n}} C^{2 n} d A\right)^{1 / 2} \leqq C^{n} \frac{T^{n}}{\sqrt{n !}} .
$$

So

$$
\sum_{n=0}^{N} 2^{n}\left\|I_{n}\left(f_{n}\right)\right\| \leqq \sum_{n=0}^{\infty} \frac{C^{\prime n}}{\sqrt{n !}}<\infty
$$

We now have all the elements needed to describe the Maassen-Meyer kernel of an Hilbert-Schmidt operator in terms of the operators $H_{M}^{E}$. Let us first detail some notations.

Let $n \leqq m \in \mathbb{N}$, we denote by $P(n, m)$ the set of the $I=\left\{i_{1}<\cdots<i_{n}\right\} \subset$ $\{1, \ldots, m\}$. For a $I \in \mathscr{P}(n, m)$, we denote by $E(I, m)=\left\{\varepsilon_{1}, \ldots, \varepsilon_{m}\right\}$ the element of $E_{m}^{*}$ such that $\varepsilon_{k}=+$ if $k \in I, \varepsilon_{k}=-$ otherwise.

Theorem 10.4. Let $H$ be an Hilbert-Schmidt operator from $\Phi$ into $\Phi$, then $H$ admits, on $\widetilde{\Xi}$, a representation as a Maassen-Meyer kernel, where the kernel $\widehat{H}$ of $H$ is given by

$$
\widehat{H}(A, B, C)=(-1)^{|B|} \sum_{I \in P(|C|,|C|+|A|)} \mathbb{1}_{\left\{S_{|C|+|A|,|A|}(I)\right\}}(C) \mathbb{E}\left[H_{A \cup C}^{E(I,|C|+|A|)} \mathbb{1}\right] .
$$

Proof. By Proposition 10.3, one has, for all $f \in \widetilde{\Xi}$, all $m \in \mathbb{N}$,

$$
\begin{aligned}
I_{m}(H f) & =\sum_{n=m}^{\infty} \sum_{\substack{E \in E_{n}^{*} \\
n_{+}(E)=m}} \int_{\mathscr{P}_{n}} \mathbb{E}\left[H_{M}^{E} \mathbb{1}\right] \mathbb{E}\left[f_{M}^{E}\right] d W_{M}^{E} \\
& =\int_{A \in \mathscr{P}_{m}}\left[\sum_{n=m}^{\infty} \sum_{\substack{E \in E_{n}^{*} \\
n_{+}(E)=m}} \int_{M \in S_{n, m}\left(I_{+}(E)\right)} \mathbb{E}\left[H_{A \cup M}^{E} \mathbb{1}\right] \mathbb{E}\left[f_{A \cup M}^{E}\right] d M\right] d W_{A} .
\end{aligned}
$$

So, if $A$ is a fixed element of $\mathscr{P}_{m}$,

$$
\begin{aligned}
\widehat{H f}(A) & =\sum_{n=0}^{\infty} \sum_{I \in P(n, n+m)} \int_{M \in \mathscr{P}_{n}} \mathbb{1}_{\left\{S_{n+m, m}(I)\right\}}(M) \mathbb{E}\left[H_{A \cup M}^{E(I, n+m)} \mathbb{1}\right] \widehat{f}(M) d M \\
& =\int_{\mathscr{P}}\left[\sum_{I \in P(|M|,|M|+m)} \mathbb{1}_{\left\{S_{|M|+m, m}(I)\right\}}(M) \mathbb{E}\left[H_{A \cup M}^{E(I,|M|+m)} \mathbb{1}\right] \widehat{f}(M)\right] d M .
\end{aligned}
$$

If one puts

$$
\varphi(A, M)=\sum_{I \in P(|M|,|M|+|A|)} \mathbb{1}_{\left\{S_{|M|+|A|,|A|}(I)\right\}}(M) \mathbb{E}\left[H_{A \cup M}^{E(I,|M|+|A|)} \mathbb{1}\right],
$$


one has proved that

$$
\widehat{H f}(A)=\int_{\mathscr{P}} \varphi(A, M) \widehat{f}(M) d M,
$$

so, using the same method as in the proof of Theorem 7.1, it is easy to see that $\widehat{H}(A, B, C)=(-1)^{|B|} \varphi(A, C)$ defines the Maassen-Meyer kernel of $H$.

The author thanks M. Emery, R.L. Hudson, J.M. Lindsay, P.-A. Meyer and the referee for their helpful corrections and comments.

\section{References}

1. Attal, S.: An algebra of non-commutative bounded semimartingales. Square and angle quantum brackets. J. Funct. Anal. 124, 292-332 (1994)

2. Attal, S., Meyer, P.-A.: Interprétation probabiliste et extension des intégrales stochastiques non commutatives. Séminaire de Probabilités XXVII, Lect. Notes in Math. 1557, Berlin, Heidelberg, New York: Springer, 1993, pp. 312-327

3. Guichardet, A.: Symmetric Hilbert spaces and related topics. Lect. Notes in Math. 261 (1970)

4. Hudson, R.L., Kree, P.: Quantum stochastic calculus for Hilbert-Schmidt processes. Stochastic analysis, path integration and dynamics, K.D. Elworthy, J.-C. Zambrini (eds.) Longman, 1989, pp. 83-93

5. Hudson, R.L., Lindsay, J.M., Parthasarathy, K.R.: Stochastic integral representation of some quantum martingales in Fock space. In: Warwick Symposium on Stochastic Differential Equations and Applications (1984/85), Pitman Research Notes on Mathematics 150, 1986, pp. $121-131$

6. Hudson, R.L., Parthasarathy, K.R.: Quantum Itô's formula and stochastic evolutions. Commun. Math. Phys. 93, 301-323 (1984)

7. Ito, K.: Multiple Wiener integral. J. Math. Soc. Japan 3, No. 1, 157-169 (1951)

8. Lindsay, J.M.: On set convolutions and integral-sum kernel operators. Proceedings: Vth Vilnius International Conference on Probability Theory and Mathematical Statistics, (1989), vol II, pp. $105-123$

9. Maassen, H.: Quantum Markov processes on Fock space described by integral kernels. Quantum Prob. and Appl. II, Lect. Notes in Math. 1136, Berlin, Heidelberg, New York: Springer, 1985, pp. 361-374

10. Meyer, P.A.: Quantum probability for probabilists. Lect. Notes in Math. 1538, Berlin, Heidelberg, New York: Springer, 1993

11. Meyer, P.A.: Eléments de probabilités quantiques. Séminaire de Probabilités XX, Lect. Notes in Math. 1204, Berlin, Heidelberg, New York: Springer, 1986, pp. 186--312

12. Meyer, P.A.: Représentation de martingales d'opérateurs. Séminaire de Probabilités XXVII, Lect. Notes in Math. 1557, Berlin, Heidelberg, New York: Springer, 1993, pp. 97-105

13. Parthasarathy, K.R.: An introduction to quantum stochastic calculus. Monographs in Mathematics, Boston, Basel: Birkhäuser, 1992

14. Parthasarathy, K.R, Sinha, K.B.: Representation of bounded quantum martingales in Fock space. J. Funct. Anal. 67, 126-151 (1986)

15. Segal, I.E.: Tensor algebras over Hilbert spaces. Trans. Am. Math. Soc. 81, 106-134 (1956)

Communicated by H. Araki 